\title{
INTEGRATION OF $E$-FUNCTIONS WITH REGARD TO THEIR PARAMETERS
}

\author{
by F. M. RAGAB
}

(Received 22nd March, 1956)

1. Introductory. In integrating $E$-functions with respect to their parameters the contours are usually of the Barnes type, deformed if necessary to separate the increasing and decreasing sequences of poles of the integrands. Also the constants are taken to be such that the integrals converge. The following formulae are required in proving the theorems given in this paper.

If $p \geqq q+1$,

$$
\begin{aligned}
& E\left(p ; \alpha_{r}: q ; \rho_{s}: z\right)=\sum_{r=1}^{p} \prod_{m=1}^{p} \Gamma\left(\alpha_{m}-\alpha_{r}\right)\left[\prod_{t=1}^{q} \Gamma\left(\rho_{t}-\alpha_{r}\right)\right]^{-1} \Gamma\left(\alpha_{r}\right) z^{\alpha_{r}} \\
& \times F\left\{\alpha_{r}, \alpha_{r}-\rho_{1}+1, \ldots, \alpha_{r}-\rho_{q}+1: \alpha_{r}-\alpha_{1}+1, \ldots * \ldots, \alpha_{r}-\alpha_{p}+1:(-1)^{p-\alpha_{z}}\right\} . \\
& E(\alpha, \beta:: z)=\Gamma(\beta) \int_{0}^{\infty} e^{-\lambda} \lambda^{\alpha-1}(1+\lambda / z)^{-\beta} d \lambda, \quad R(\alpha)>0 . \\
& E\left(p ; \alpha_{r}: q ; \rho_{s}: z\right)=\frac{1}{2 \pi i} \int \frac{\Gamma(\zeta) \stackrel{p}{\prod} \Gamma\left(\alpha_{r}-\zeta\right)}{\prod_{t=1}^{q} \Gamma\left(\rho_{t}-\zeta\right)} z^{\zeta} d \zeta,
\end{aligned}
$$

the contour being of the Barnes type.

$$
\begin{aligned}
& \frac{1}{2 \pi i} \int \Gamma(\alpha-\zeta) \Gamma(\beta-\zeta) \Gamma(\gamma+\zeta) \Gamma(\delta+\zeta) z^{\zeta} d \zeta \\
&=\sum_{\alpha, \beta} \Gamma(\beta-\alpha) \Gamma(\alpha+\gamma) \Gamma(\alpha+\delta) z^{\alpha} F(\alpha+\gamma, \alpha+\delta ; \alpha-\beta+1 ; z) .
\end{aligned}
$$

This result can be obtained by evaluating the residues at the poles of the integrand.

$$
\begin{aligned}
& F(\alpha, \beta ; \gamma ; z)=\frac{\Gamma(\gamma-\alpha-\beta) \Gamma(\gamma)}{\Gamma(\gamma-\alpha) \Gamma(\gamma-\beta)} F\left(\begin{array}{c}
\alpha, \beta . \\
\alpha+\beta-\gamma+1
\end{array} ; 1-z\right) \\
& +\frac{\Gamma(\alpha+\beta-\gamma) \Gamma(\gamma)}{\Gamma(\alpha) \Gamma(\beta)}(1-z)^{\gamma-\alpha-\beta} F\left(\begin{array}{l}
\gamma-\alpha, \gamma-\beta ; 1-z \\
\gamma-\alpha-\beta+1
\end{array}\right) . \\
& E\left(\frac{1}{2}-k+m, \frac{1}{2}-k-m:: z\right)=\Gamma\left(\frac{1}{2}-k+m\right) \Gamma\left(\frac{1}{2}-k-m\right) z^{-k} e^{\frac{1}{2} z} W_{k, m}(z) \text {. } \\
& \cos n \pi E\left(\frac{1}{2}+n, \frac{1}{2}-n:: 2 z\right)=\sqrt{ }(2 \pi z) e^{z} K_{n}(z)
\end{aligned}
$$

For these formulae see MacRobert's Functions of a Complex Variable, 4th edition.

2. Theorem 1. The theorem to be proved is

$$
\frac{1}{2 \pi i} \int E(\alpha+\zeta, \gamma:: z) E(\beta-\zeta, \delta:: z) d \zeta=2^{-\alpha-\beta} \frac{\Gamma(\gamma) \Gamma(\delta)}{\Gamma(\gamma+\delta)} E(\alpha+\beta, \gamma+\delta:: 2 z) \text {, }
$$

where the integral is of the Barnes type.

To prove this, substitute for each $E$-function in the integral from (2) and change the 
order of integration, so getting

$$
\begin{aligned}
& \int_{0}^{\infty} \int_{0}^{\infty} e^{-\lambda-\mu} \lambda^{\nu-1} \mu^{\delta-1}(1+\lambda / z)^{-\alpha}(1+\mu / z)^{-\beta} d \lambda d \mu \frac{1}{2 \pi i} \int(1+\lambda / z)^{-\zeta}(1+\mu / z)^{\zeta} \Gamma(\alpha+\zeta) \Gamma(\beta-\zeta) d \zeta \\
& =\int_{0}^{\infty} \int_{0}^{\infty} e^{-\lambda-\mu} \lambda^{\gamma-1} \mu^{8-1}(1+\mu / z)^{-\alpha-\beta} E\left(\alpha+\beta:: \frac{1+\mu / z}{1+\lambda / z}\right) d \lambda d \mu, \quad \text { by (3). }
\end{aligned}
$$

Thus, since

$$
E(\alpha:: z)=\Gamma(\alpha)(1+1 / z)^{-\alpha}
$$

the left hand side is equal to

$$
2^{-\alpha-\beta} \Gamma(\alpha+\beta) \int_{0}^{\infty} \int_{0}^{\infty} e^{-\lambda-\mu} \lambda^{\gamma-1} \mu^{\delta-1}\left(1+\frac{\lambda+\mu}{2 z}\right)^{-\alpha-\beta} d \lambda d \mu .
$$

Here put $\lambda=\mu \eta$, and it becomes

$$
\begin{aligned}
2^{-\alpha-\beta} \Gamma(\alpha+\beta) \int_{0}^{\infty} \int_{0}^{\infty} e^{-\mu(1+\eta)} \mu^{\nu+\delta-1} \eta^{\gamma-1}\{1+\mu(1+\eta) /(2 z)\}^{-\alpha-\beta} d \mu d \eta \\
\quad=2^{-\alpha-\beta} \Gamma(\alpha+\beta) \int_{0}^{\infty} \frac{\eta^{\gamma-1}}{(1+\eta)^{\gamma+\delta}} d \eta \int_{0}^{\infty} e^{-\mu} \mu^{\gamma+\delta-1}\left(1+\frac{\mu}{2 z}\right)^{-\alpha-\beta} d \mu
\end{aligned}
$$

and from this, on applying (2), the result is obtained.

In particular, if $\beta=\frac{1}{2}+n-\alpha, \delta=\frac{1}{2}-n-\gamma$, it follows from (7) that

$\frac{1}{2 \pi i} \int E(\alpha+\zeta, \gamma:: z) E\left(\frac{1}{2}+n-\alpha-\zeta, \frac{1}{2}-n-\gamma:: z\right) d \zeta$

$$
=2^{-n} \sqrt{ }(z / \pi) \Gamma(\gamma) \Gamma\left(\frac{1}{2}+n\right) \Gamma\left(\frac{1}{2}-n-\gamma\right) e^{z} K_{n}(z) .
$$

3. Theorem 2. The formula to be proved is

$$
\begin{aligned}
\frac{1}{2 \pi i} \int E(\alpha & +\zeta, \beta+\zeta:: z) E(\gamma-\zeta, \delta-\zeta:: z) d \zeta \\
& =2^{1-\alpha-\beta-\gamma-\delta} \sqrt{ } \pi E\left\{\alpha+\gamma, \alpha+\delta, \beta+\gamma, \beta+\delta: \frac{1}{2}(\alpha+\beta+\gamma+\delta), \frac{1}{2}(\alpha+\beta+\gamma+\delta+1): 2 z\right\} .
\end{aligned}
$$

To prove this, substitute from (1) for each $E$-function on the left and get

$$
\begin{array}{r}
\sum_{\alpha, \beta} \sum_{\gamma, \delta} \Gamma(\beta-\alpha) \Gamma(\delta-\gamma) \sum_{m=0}^{\infty} \sum_{n=0}^{\infty} \frac{z^{\alpha+\gamma+m+n}}{m ! n !(\alpha-\beta+1 ; m)(\gamma-\delta+1 ; n)} \\
\quad \times \frac{1}{2 \pi i} \int \Gamma(\alpha+m+\zeta) \Gamma(\gamma+n-\zeta) d \zeta .
\end{array}
$$

Now, from (3), the last line is equal to

$$
E(\alpha+\gamma+m+n:: 1)=\Gamma(\alpha+\gamma+m+n) 2^{-\alpha-\gamma-m-n} .
$$

Hence the above expression is equal to

$$
\begin{aligned}
& \sum_{\alpha, \beta} \sum_{\gamma, \delta} \Gamma(\alpha+\gamma) \Gamma(\beta-\alpha) \Gamma(\delta-\gamma) \sum_{m=0}^{\infty} \sum_{n=0}^{\infty} \frac{(\alpha+\gamma ; m+n)\left(\frac{1}{2} z\right)^{\alpha+\gamma+m+n}}{m ! n !(\alpha-\beta+1 ; m)(\gamma-\delta+1 ; n)} \\
& \quad=\sum_{\alpha, \beta} \sum_{\gamma, \delta} \Gamma(\alpha+\gamma) \Gamma(\beta-\alpha) \Gamma(\delta-\gamma)\left(\frac{1}{2} z\right)^{\alpha+\gamma} \sum_{p=0}^{\infty} \frac{(\alpha+\gamma ; p)\left(\frac{1}{2} z\right)^{p}}{p !(\gamma-\delta+1 ; p)} \sum_{m=0}^{p} \frac{(-p ; m)(\delta-\gamma-p ; m)}{m !(\alpha-\beta+1 ; m)} .
\end{aligned}
$$

On applying Gauss's theorem to the last summation it becomes

$$
\frac{\Gamma(\alpha-\beta+1) \Gamma(\alpha-\beta+\gamma-\delta+1+2 p)}{\Gamma(\alpha-\beta+1+p) \Gamma(\alpha-\beta+\gamma-\delta+1+p)},
$$


and therefore the given expression is equal to

$\sum_{\alpha, \beta} \sum_{\gamma, \delta} \Gamma(\beta-\alpha) \Gamma(\delta-\gamma) \Gamma(\alpha+\gamma)\left(\frac{1}{2} z\right)^{\alpha+\gamma} F\left\{\begin{array}{c}\alpha+\gamma, \frac{1}{2}(\alpha-\beta+\gamma-\delta+1), \frac{1}{2}(\alpha-\beta+\gamma-\delta+2) ; 2 z \\ \alpha-\beta+1, \gamma-\delta+1, \alpha-\beta+\gamma-\delta+1\end{array}\right\}$

From (1) the result then follows.

In particular, if $\gamma=\alpha, \delta=\beta$,

$\frac{1}{2 \pi i} \int E(\alpha+\zeta, \beta+\zeta:: z) E(\alpha-\zeta, \beta-\zeta:: z) d \zeta=2^{1-2 \alpha-2 \beta} \sqrt{ } \pi E\left(2 \alpha, 2 \beta, \alpha+\beta: \alpha+\beta+\frac{1}{2}: 2 z\right)$.

4. Theorem 3. The theorem to be proved is

$$
\begin{aligned}
\frac{1}{2 \pi i} \int \Gamma(\alpha+\zeta) \Gamma(\beta+\zeta) \Gamma(\gamma-\zeta) E\left(\delta-\zeta, \alpha_{1}, \ldots, \alpha_{p}: q ; \rho_{s}: z\right) d \zeta \\
=\Gamma(\alpha+\gamma) \Gamma(\beta+\gamma) E\left(\alpha+\delta, \beta+\delta, \alpha_{1}, \ldots, \alpha_{p}: \alpha+\beta+\gamma+\delta, \rho_{1}, \ldots, \rho_{q}: z\right) .
\end{aligned}
$$

To prove this, consider the case $p=q=0$; then the left hand side can be written

$$
\frac{(1+1 / z)^{-8}}{2 \pi i} \int \Gamma(\alpha+\zeta) \Gamma(\beta+\zeta) \Gamma(\gamma-\zeta) \Gamma(\delta-\zeta)\left(1+\frac{1}{z}\right)^{\zeta} d \zeta
$$

and, from (4), this is equal to

$$
\left(1+\frac{1}{z}\right)^{-\delta} \underset{\gamma, \delta}{\Sigma} \Gamma(\gamma-\delta) \Gamma(\alpha+\delta) \Gamma(\beta+\delta)\left(1+\frac{1}{z}\right)^{\delta} F\left(\alpha+\delta, \beta+\delta ; \delta-\gamma+1 ; 1+\frac{1}{z}\right)
$$

provided that $\left|1+\frac{1}{z}\right|<1$. From (5) it follows that this is equal to

$$
\begin{aligned}
\frac{\Gamma(\alpha+\gamma) \Gamma(\beta+\gamma) \Gamma(\alpha+\delta) \Gamma(\beta+\delta)}{\Gamma(\alpha+\beta+\gamma+\delta)} F\left(\begin{array}{c}
\alpha+\delta, \beta+\delta \\
\alpha+\beta+\gamma+\delta
\end{array} ;-1 / z\right) \\
=\Gamma(\alpha+\gamma) \Gamma(\beta+\gamma) E(\alpha+\delta, \beta+\delta: \alpha+\beta+\gamma+\delta: z),
\end{aligned}
$$

and the result can then be deduced by generalising.

In particular, if $p=1, q=2, \alpha_{1}=\alpha+\beta+\gamma+\delta, \rho_{1}=\alpha+\delta, \rho_{2}=\beta+\delta$,

$\frac{1}{2 \pi i} \int \Gamma(\alpha+\zeta) \Gamma(\beta+\zeta) \Gamma(\gamma-\zeta) \Gamma(\delta-\zeta) F(\alpha+\beta+\gamma+\delta, \delta-\zeta: \alpha+\delta, \beta+\delta:-1 / z) d \zeta$

$$
=\Gamma(\alpha+\gamma) \Gamma(\beta+\gamma) \Gamma(\alpha+\delta) \Gamma(\beta+\delta)\{\Gamma(\alpha+\beta+\gamma+\delta)\}^{-1} \exp (-1 / z) .
$$

5. Theorem 4. The theorem to be proved is

$$
\begin{aligned}
& \frac{1}{2 \pi i} \int \Gamma(\gamma+\zeta) \Gamma(\delta+\zeta) E(\alpha-\zeta, \beta-\zeta:: z) d \zeta \\
& \quad=2^{1-\alpha-\beta-\gamma-\delta} \sqrt{\pi} E\left\{\alpha+\gamma, \beta+\gamma, \alpha+\delta, \beta+\delta: \frac{1}{2}(\alpha+\beta+\gamma+\delta), \frac{1}{2}(\alpha+\beta+\gamma+\delta+1): 4 z\right\} .
\end{aligned}
$$

To prove this, substitute for the $E$-function on the left from (1) and get

$$
\begin{aligned}
& \sum_{\alpha, \beta} \Gamma(\beta-\alpha) \sum_{m=0}^{\infty} \frac{z^{\alpha+m}}{m !(\alpha-\beta+1 ; m)} \frac{1}{2 \pi i} \int \Gamma(\gamma+\zeta) \Gamma(\delta+\zeta) \Gamma(\alpha+m-\zeta) z^{-\zeta} d \zeta \\
& =\sum_{\alpha, \beta} \Gamma(\beta-\alpha) \sum_{m=0}^{\infty} \frac{1}{m !(\alpha-\beta+1 ; m)} E(\alpha+\gamma+m, \alpha+\delta+m:: z) \\
& =\sum_{\alpha, \beta} \sum_{\gamma, \delta} \Gamma(\beta-\alpha) \Gamma(\delta-\gamma) \sum_{m=0}^{\infty} \sum_{n=0}^{\infty} \frac{\Gamma(\alpha+\gamma+m+n) z^{\alpha+\gamma+m+n}}{m ! n !(\alpha-\beta+1 ; m)(\gamma-\delta+1 ; n)} \\
& =\sum_{\alpha, \beta} \sum_{\gamma, \delta} \Gamma(\beta-\alpha) \Gamma(\delta-\gamma) \Gamma(\alpha+\gamma) z^{\alpha+\gamma} \sum_{p=0}^{\infty} \frac{(\alpha+\gamma ; p) z^{p}}{p !(\gamma-\delta+1 ; p)} F\left(\begin{array}{c}
-p, \delta-\gamma-p ; 1 \\
\alpha-\beta+1
\end{array}\right)
\end{aligned}
$$


$=\sum_{\alpha, \beta} \sum_{\gamma, \delta} \Gamma(\beta-\alpha) \Gamma(\delta-\gamma) \Gamma(\alpha+\gamma) z^{\alpha+\gamma} F\left\{\begin{array}{c}\alpha+\gamma, \frac{1}{2}(\alpha-\beta+\gamma-\delta+1), \frac{1}{2}(\alpha-\beta+\gamma-\delta+2) ; 4 z \\ \alpha-\beta+1, \gamma-\delta+1, \alpha-\beta+\gamma-\delta+1\end{array}\right\}$,

and from this, using (1), the result follows.

In particular, if $\gamma=\alpha, \delta=\beta$,

$$
\begin{aligned}
\frac{1}{2 \pi i} \int \Gamma(\alpha+\zeta) \Gamma(\beta+\zeta) E(\alpha & -\zeta, \beta-\zeta:: z) d \zeta \\
& =2^{1-2 \alpha-2 \beta} \sqrt{ } \pi E\left(2 \alpha, 2 \beta, \alpha+\beta: \alpha+\beta+\frac{1}{2}: 4 z\right) .
\end{aligned}
$$

6. Theorem 5. The theorem to be proved is

$$
\begin{aligned}
& \frac{1}{2 \pi i} \int \Gamma(\alpha-\zeta) \Gamma(\beta+\zeta) E(\gamma-\zeta, \delta+\zeta:: z) d \zeta \\
& \quad=\Gamma(\alpha+\beta) 2^{-\alpha-\beta} E\left\{\alpha+\delta, \beta+\gamma, \frac{1}{2}(\gamma+\delta), \frac{1}{2}(\gamma+\delta+1): \frac{1}{2}(\alpha+\beta+\gamma+\delta), \frac{1}{2}(\alpha+\beta+\gamma+\delta+1): z\right\} .
\end{aligned}
$$

The following two formulae are required in the proof.

If $R(\alpha)>0, R(\beta)>0$,

$$
\begin{aligned}
\int_{0}^{\infty} \lambda^{\alpha-1}(1+\lambda)^{-\alpha-\beta} & E\left(p ; \alpha_{r}: q ; \rho_{s}: z(1+\lambda)^{2} / \lambda\right\} d \lambda \\
= & 2^{1-\alpha-\beta} \sqrt{ } \pi E\left\{\alpha, \beta, \alpha_{1}, \ldots, \alpha_{p}: \frac{1}{2}(\alpha+\beta), \frac{1}{2}(\alpha+\beta+1), \rho_{1}, \ldots, \rho_{a}: 4 z\right\} .
\end{aligned}
$$

If $m$ is a positive integer and if $R(k)>0$,

$$
\int_{0}^{\infty} e^{-\lambda} \lambda^{k-1} E\left(p ; \alpha_{r}: q ; \rho_{s}: z / \lambda^{m}\right) d \lambda=m^{k-1}(2 \pi)^{\frac{1}{d} m} E\left(p+m ; \alpha_{r}: q ; \rho_{s}: z / m^{m}\right),
$$

where $\alpha_{p+1+\nu}=(k+\nu) / m, \nu=0,1,2, \ldots, m-1$.

For (17) consider the case $p \leqq q+1$, expand in powers of $z$ and integrate term by term. Then generalise to get the case $p>q+1$.

Now, from (2), if $R(\alpha)>0, R(\beta)>0$,

$$
E(\alpha, \beta:: z)=\int_{0}^{\infty} e^{-\lambda} \lambda^{\alpha-1} d \lambda \int_{0}^{\infty} e^{-\mu(1+\lambda / z)} \mu^{\beta-1} d \mu .
$$

Hence, on substituting in the left hand side of (16), and changing the order of integration, it becomes

$$
\begin{aligned}
\int_{0}^{\infty} e^{-\lambda} \lambda^{\gamma-1} d \lambda \int_{0}^{\infty} e^{-\mu(1+\lambda / z)} \mu^{\delta-1} d \mu \frac{1}{2 \pi i} \int \Gamma(\alpha-\zeta) \Gamma(\beta+\zeta)(\mu / \lambda)^{\zeta} d \zeta \\
=\int_{0}^{\infty} e^{-\lambda \lambda^{\gamma-1}} d \lambda \int_{0}^{\infty} e^{-\mu(1+\lambda / z)} \mu^{\delta-1}(\mu / \lambda)^{-\beta} E(\alpha+\beta:: \mu / \lambda) d \mu .
\end{aligned}
$$

Here replace $\mu$ by $\mu \lambda$, change the order of integration, and get

$$
\begin{aligned}
\int_{0}^{\infty} \mu^{\delta-\beta-1} E(\alpha+\beta:: \mu) d \mu \int_{0}^{\infty} e^{-\lambda(1+\mu)} \lambda^{\gamma+\delta-1} E\left(:: \frac{z}{\lambda^{2} \mu}\right) d \lambda \\
\quad=\int_{0}^{\infty} \mu^{\delta-\beta-1}(1+\mu)^{-\gamma-\delta} E(\alpha+\beta:: \mu) d \mu \int_{0}^{\infty} e^{-\lambda} \lambda^{\gamma+\delta-1} E\left\{:: \frac{z(1+\mu)^{2}}{\lambda^{2} \mu}\right\} d \lambda \\
=2^{\gamma+\delta-1} \pi^{-1} \Gamma(\alpha+\beta) \int_{0}^{\infty} \mu^{\alpha+\delta-1}(1+\mu)^{-\alpha-\beta-\gamma-\delta} E\left\{\frac{\gamma+\delta}{2}, \frac{\gamma+\delta+1}{2}:: \frac{z(1+\mu)^{2}}{4 \mu}\right\},
\end{aligned}
$$


by (18). On applying (17) the right hand side is obtained.

For example if, in (16), $\gamma=\frac{1}{2}-k+m, \delta=\frac{1}{2}-k-m$, then, from (6),

$$
\begin{aligned}
\frac{1}{2 \pi i} \int \Gamma(\alpha-\zeta) \Gamma(\beta+\zeta) \Gamma\left(\frac{1}{2}-k+m-\zeta\right) \Gamma\left(\frac{1}{2}-k-m+\zeta\right) W_{k, m-\zeta}(z) d \zeta \\
\quad=2^{-\alpha-\beta} \Gamma(\alpha+\beta) z^{k} e^{-\frac{1}{2} z} E\left(\begin{array}{l}
\alpha+\frac{1}{2}-k-m, \beta+\frac{1}{2}-k+m, \frac{1}{2}-k, 1-k: z \\
\frac{1}{2}-k+\frac{1}{2} \alpha+\frac{1}{2} \beta, 1-k+\frac{1}{2} \alpha+\frac{1}{2} \beta
\end{array}\right) .
\end{aligned}
$$

7. Theorem 6. The theorem to be proved is

$$
\begin{aligned}
\frac{1}{2 \pi i} \int E\left(\alpha+\zeta, \beta-\zeta, \alpha_{1}, \ldots\right. & \left., \alpha_{p}: q ; \rho_{s}: z\right) d \zeta \\
& =\frac{1}{2} \pi^{-\frac{1}{2}} E\left(\frac{1}{2} \alpha+\frac{1}{2} \beta, \frac{1}{2} \alpha+\frac{1}{2} \beta+\frac{1}{2}, \alpha_{1}, \ldots, \alpha_{\nu}: q ; \rho_{s}: z\right) .
\end{aligned}
$$

To prove this, consider the case in which $p=q=0$, substitute for the $E$-function on the left from (2) and change the order of integration; then the left hand side becomes

$$
\begin{aligned}
& \int_{0}^{\infty} e^{-\lambda} \lambda^{\beta-1}(1+\lambda / z)^{-\alpha} d \lambda \frac{1}{2 \pi i} \int \Gamma(\alpha+\zeta) \lambda^{-\zeta}(1+\lambda / z)^{-\zeta} d \zeta \\
&=\int_{0}^{\infty} e^{-\lambda} \lambda^{\alpha+\beta-1} \exp \left(-\lambda-\lambda^{2} / z\right) d \lambda=2^{-\alpha-\beta} \int_{0}^{\infty} e^{-\mu} \mu^{\alpha+\beta-1} E\left(:: 4 z \mu^{-2}\right) d \mu \\
&=\frac{1}{2} \pi^{-\frac{1}{2}} E\left(\frac{1}{2} \alpha+\frac{1}{2} \beta, \frac{1}{2} \alpha+\frac{1}{2} \beta+\frac{1}{2}:: z\right) .
\end{aligned}
$$

Now generalise and obtain (21).

8. Theorem 7. The theorem to be proved is

$$
\begin{aligned}
& \frac{1}{2 \pi i} \int \Gamma(\alpha+\zeta) \Gamma(\beta-\zeta) E\left(p ; \alpha_{r}-\zeta: k-2 \zeta, \rho_{1}-\zeta, \ldots, \rho_{q}-\zeta: e^{ \pm i \pi} z\right) z^{\zeta} d \zeta \\
& \quad=2^{-\alpha-\beta} \Gamma(\alpha+\beta) z^{-\alpha} E\left(\begin{array}{l}
\frac{1}{2} k+\frac{1}{2} \alpha-\frac{1}{2} \beta, \frac{1}{2}+\frac{1}{2} k+\frac{1}{2} \alpha-\frac{1}{2} \beta, \alpha_{1}+\alpha, \ldots, \alpha_{p}+\alpha: e^{ \pm i \pi} z \\
\frac{1}{2} k+\alpha, \frac{1}{2}+\frac{1}{2} k+\alpha, k+\alpha-\beta, \rho_{1}+\alpha, \ldots, \rho_{q}+\alpha
\end{array}\right) .
\end{aligned}
$$

To prove it, substitute on the left from formula (3) and so obtain

$$
\frac{1}{2 \pi i} \int \Gamma(\alpha+\zeta) \Gamma(\beta-\zeta) z^{\zeta} d \zeta \frac{1}{2 \pi i} \int \frac{\Gamma(Z) \Pi \Gamma\left(\alpha_{r}-\zeta-Z\right)}{\Gamma(k-2 \zeta-Z) \Gamma\left(\rho_{s}-\zeta-Z\right)}\left(e^{ \pm i \pi} z\right)^{Z} d Z .
$$

Here replace $Z$ by $Z-\zeta$ and change the order of integration, so getting

$$
\frac{1}{2 \pi i} \int \frac{\Pi \Gamma\left(a_{r}-Z\right)}{\Pi \Gamma\left(\rho_{s}-Z\right)}\left(e^{ \pm i \pi} z\right)^{Z} d Z \frac{1}{2 \pi i} \int \frac{\Gamma(\alpha+\zeta) \Gamma(\beta-\zeta) \Gamma(Z-\zeta)}{\Gamma(k-Z-\zeta)} e^{\mp i \pi \zeta} d \zeta
$$

Now the inner integral is equal to

$$
e^{ \pm i \pi \alpha} E\left(\alpha+\beta, \alpha+Z: k+\alpha-Z: e^{\mp i \pi}\right),
$$

and, by Gauss's Theorem, this $E$-function has the value

$$
\frac{\Gamma(\alpha+\beta) \Gamma(\alpha+Z) \Gamma(k-\alpha-\beta-2 Z)}{\Gamma(k-\beta-Z) \Gamma(k-2 Z)} .
$$

Hence, on applying the duplication formula for the gamma function and formula (3), the right hand side is obtained.

Faculty of Science

EIN Shams UNIVERgITY

Catro 